\title{
Management of a Patient with Double Aortic Arch and Severe Tracheal Compression
}

\author{
Aris Lacis, Inta Bergmane, Elina Ligere, Valts Ozolins, Lauris Smits, Normunds Sikora \\ Children`s University Hospital, Department of Pediatric Cardiology and Cardiac Surgery, Riga, Latvia
}

\begin{abstract}
Summary
Vascular ring presents less than $1 \%$ of all congenital cardiovascular anomalies and may cause respiratory symptoms and feeding problems, double aortic arch (DAA) is the most common vascular ring (40\%). The anomaly is due to a failure of regression of both the right and the left fourth branchial arches, resulting in right and left aortic arches which completely encircle and compress the trachea and esophagus, producing respiratory distress and feeding problems in early infancy $(1,2,3,4)$. The first post-mortem description of double aortic arch was in 1737 by Hommell. In 1945 Gross accomplished the first successful surgical intervention for a DAA (3). Double aortic arch is commonly an isolated anomaly but is occasionally associated with a variety of congenital heart defects (ventricular septal defect, transposition of the great arteries, tetrology of Fallot, coarctation of the aorta and chromosomal abnormalities as microdeletion of 22q11.2. The clinical course of the disease and its manifestation depends on the compression of the esophagus and trachea. The most common symptoms are persistent cough $(78 \%)$, dyspnoe $(75 \%)$, recurrent respiratory infections $(56 \%)$, feeding problems $(25-40 \%)$, and failure to thrive $(5 \%)$. Symptomatic tracheobronchial compression varies inversely with the severity of compression. Tracheal compression causes airflow obstruction and decreased mucociliary clearance of secretions, leading to recurrent bronchopulmonary infections. Reflex apnoe is hypothesized to be a type of respiratory arrest that occurs when vagal afferent nerves are stimulated. Respiratory distress and a history of recurrent pulmonary infections and apneic spells are indications for surgical intervention $(1,2,3)$. Division of the smaller of the two arches (usually the left) is performed through the left thoracotomy. There are three successful operations in the cases of vascular ring done in the Clinics for Pediatric cardiology and Cardiac surgery of University hospital for Children in Riga. We present the one of the cases with successful treatment of severe tracheal compression.
\end{abstract}

Key words: double aortic arch, vascular rings, congenital cardiac disease, cardiovascular surgery.

\section{AIM OF THE DEMONSTRATION}

Aim of the demonstration is to show the complete resolution of severe tracheal compression after the surgical treatment of double aortic arch.

\section{CASE REPORT}

The female patient $\mathrm{N}$. was first hospitalized at the age of two months due to obstructive bronchitis, inspiratory and expiratory stridor. The child was born to healthy mother from the $5^{\text {th }}$ pregnancy, $3^{\text {rd }}$ delivery at the gestation age of 37 weeks, birth weight $2850 \mathrm{~g}$. She was breastfed for two weeks and afterwards received adapted formula. The child had frequent regurgitations, wheezing was observed following feeding. At the admission to the hospital at the age of two months weight was 5300g, some disembriogenic stigmata were observed- hypertelorism, small ears. The heart rate was 156 times per minute with systolic murmur grade 3/6 with maximum at the left 3 rd intercostal space, normal femoral pulses, no hepatomegaly present. The child was breathing with a use of accessory muscles, prolonged expiration and dry crackles auscultated. The direct laryngoscopy showed the signs of acute laryngitis. The thoracic X-ray showed the small shadow of glandula thymus, no signs of congestion and the cardio-thoracic coefficient was 0,59. Electrocardiogram was consistent with the patient's age -synus rhythm 166 times per minute while baby crying. Echocardiography revealed perimembranous ventricular septal defect $5 \mathrm{~mm}$ in size with a left to right shunt with pressure gradient $92 \mathrm{mmHg}$, LVDd $22 \mathrm{~mm}$, fractional shortening (FS) $40 \%$, ejection fraction $>60 \%$, aortic valve $8 \mathrm{~mm}$, pulmonary artery $10 \mathrm{~mm}$, patent foramen ovale $3 \mathrm{~mm}$. Neurosonography and abdominal ultrasound examination were without pathological changes. Clinical analyses were within the normal range. 24- Hour ph-metry showed 1, 5\% total number of refluxes. The child had normal karyothype, microdeletion of chromosome 22q11.2 excluded. During the hospital stay she received inhalations with Salbutamol, Budesonide and Furosemide, Verospirone, Captopril as a treatment of cardio-vascular insufficiency. The baby was fed with formula Aptamil pepti with rice flour addition. The girl was discharged from the hospital with diagnoses: congenital heart disease, ventricular septal defect, cow's milk protein intolerance, gastro-esophageal reflux, reflux laryngitis, obstructive bronchitis. At home the baby received the same therapy of cardio-vascular insufficiency, inhalations, omeprazol. At the next visit to paediatric cardiologist mother complained that the child's condition has not improved and the wheezing is still observed. The child was admitted to the hospital for the further examination at the age of 4 months. The contrast $x$-ray of the esophagus showed slight compression of the esophagus 
at the level of the $4^{\text {th }}$ thoracic vertebra. The computed tomography with angiography due to double aortic arch suspected was performed. It revealed the double aortic arch with severe tracheal compression (the size of trachea at the narrowest point was $1,8 \mathrm{~mm}$ ), the size of both arches was $1: 1$. Because of the severe tracheal compression the bronchoscopy was performed which showed normal larynx and unchanged vocal cords, 2/3 of the upper tracheal lumen was free and the tracheal mucous tissues without the signs of inflammation. The tracheal lumen was fissure-like $2 \mathrm{~cm}$ above the bifurcation, compression from the outside suspected, the length of the compressed segment was $1,5 \mathrm{~cm}$ and it was possible to cross it with the bronchoscope. The operation: left thoracotomy, division of the double aortic arch (division of the left arch) was performed at the age of 5 months. The bronchoscopy following the operation showed the deformation of the tracheal lumen but it was easy to cross with the bronchoscope. Repeated computed tomography 6 months after the operation showed almost complete resolution of the tracheal compression. The child develops normally, no symptoms of wheezing observed, the ventricular septal defect closed spontaneously and the therapy was discontinued.

\section{DISCUSSION}

Double aortic arch is a rare congenital heart disease although the most frequently observed vascular ring that completely encircles trachea and esophagus. Remarkable history of respiratory distress, postprandial choking since birth, dysphagia and recurrent infections of lower respiratory tract are noted in majority of patients with double aortic arch $(1,2)$. In every infant with wheezing, stridor and disphagia the presence of a double aortic arch should be carefully ruled out. Barium esophagogram is a valuable investigation which frequently shows indentation of the esophagus in the case of a vascular ring and can be safely and rapidly performed. Echocardiography is very useful to identify the associated congenital heart defects but the definite diagnosis of a double aortic arch is not easy because only subcostal and suprasternal views offer diagnostic windows for identifying a double aortic arch. For sufficient evaluation of the patient prior to surgery computed tomography (CT) or magnetic resonance imaging (MRI) is performed to improve the outcome through the ability to plan the operative strategy. Preoperative bronchoscopy can be performed to rule out associated tracheomalacia and bronchomalacia and is important for long and shot term prognosis. Outcomes are excellent after repair of double aortic arch, although persistent respiratory symptoms are frequent and probably associated with previous compression-related maldevelopment of the trachea and major airways.

Early diagnosis and surgery are important to reduce the long-term sequel of tracheobronchial compression in children.

\section{Conflict of interest: None}

\section{REFERENCES}

1. Alsenaidi K, Gurofsky R, Karamlou T, Williams WG, McCrindle BW. Management and Outcomes of Double Aortic Arch in 81 Patients// Pediatrics, 2006; 118:1336-1341

2. Park MK, Vascular Ring// In: Pediatric Cardiology for Practitioners. 5th ed. USA, Mosby Elsevier; 2008; 303-308

3. Shanmugam G, Macarthur K, Pollock J. Surgical Repair of Double Aortic Arch: 16-year Experience // Asian Cardiovasc Thorac Ann, 2005; 13:4-10

4. Weinberg PM, Aortic arch anomalies, Double Aortic Arch // In: Heart Disease in Infants, Children, and Adolescents. 7th ed. USA, Moss and Adams, Lippincott Williams and Wilkins, 2008, Volume 1, 749-752

\section{Address:}

Elina Ligere

Children`s University Hospital,

Department of Pediatric Cardiology and Cardiac

Surgery

Vienības gatve 45, LV-1004,

Riga, Latvia

E-mail: eteivane@inbox.lv 


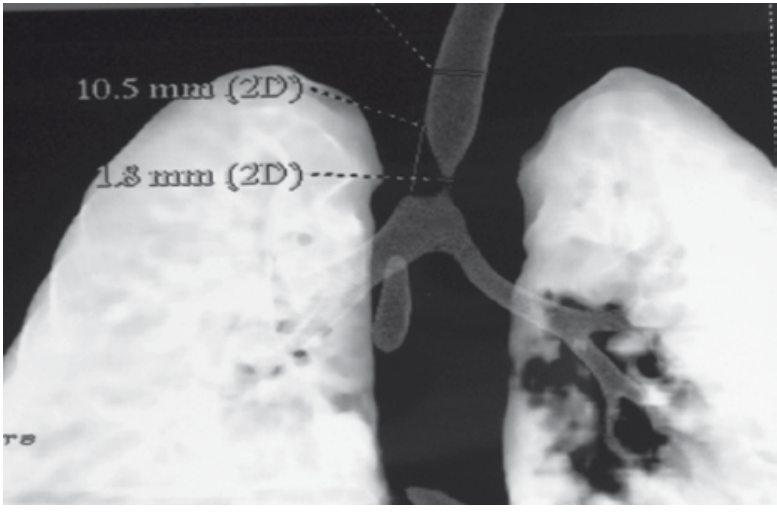

Fig. 1. Computed tomography at the age of 4 months: severe tracheal compression due to a double aortic arch

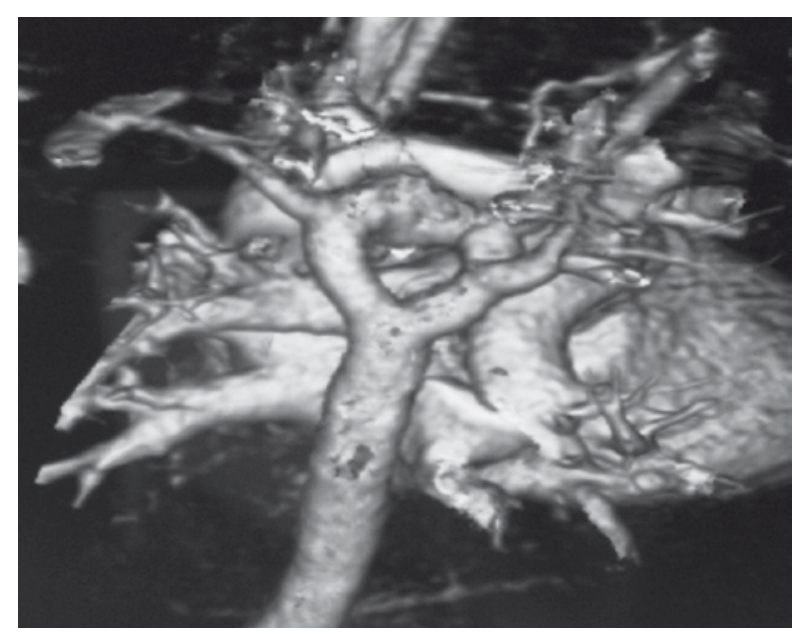

Fig. 2. CT: a double aortic arch (the size of both arches 1:1)

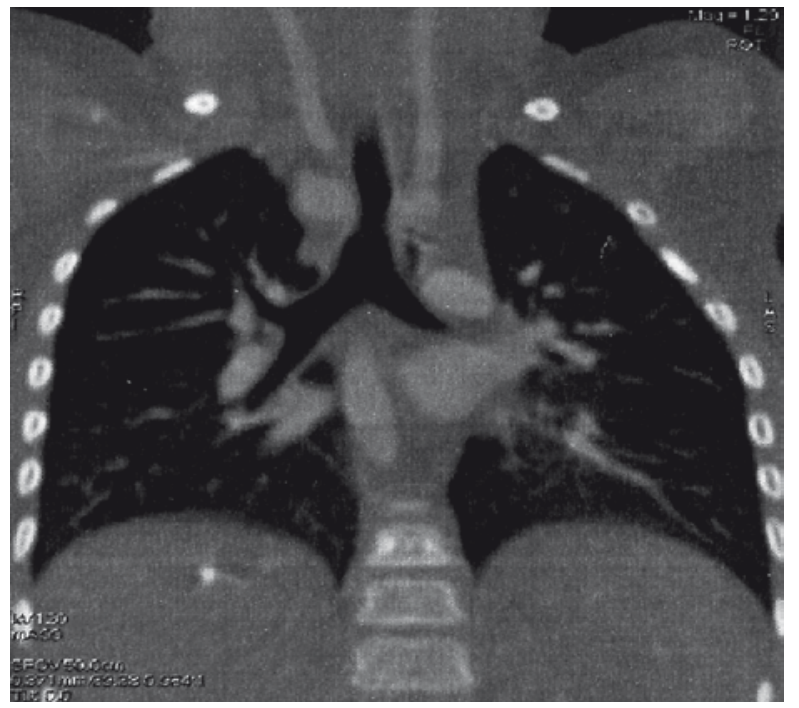

Fig. 3. Repeated computed tomography 6 months after the operation- almost complete resolution of the tracheal compression 